$6-25-2018$

\title{
Compensation for Inertial and Gravity Effects in a Moving Force Platform
}

\author{
Sandra K. Hnat \\ Cleveland State University \\ Ben J.H. van Basten \\ Motekforce Link
}

Antonie J. van den Bogert

Cleveland State University, a.vandenbogert@csuohio.edu

Follow this and additional works at: https://engagedscholarship.csuohio.edu/enme_facpub

Part of the Biomechanics and Biotransport Commons, and the Mechanical Engineering Commons How does access to this work benefit you? Let us know!

\section{Recommended Citation}

Hnat, Sandra K.; van Basten, Ben J.H.; and van den Bogert, Antonie J., "Compensation for Inertial and Gravity Effects in a Moving Force Platform" (2018). Mechanical Engineering Faculty Publications. 325. https://engagedscholarship.csuohio.edu/enme_facpub/325

This Article is brought to you for free and open access by the Mechanical Engineering Department at EngagedScholarship@CSU. It has been accepted for inclusion in Mechanical Engineering Faculty Publications by an authorized administrator of EngagedScholarship@CSU. For more information, please contact library.es@csuohio.edu. 


\title{
Compensation for Inertial and Gravity Effects in a Moving Force Platform
}

\author{
Sandra K. Hnat ${ }^{\mathrm{a}, *}$, Ben J. H. van Basten ${ }^{\mathrm{b}}$, Antonie J. van den Bogert ${ }^{\mathrm{a}}$ \\ ${ }^{a}$ Cleveland State University, Department of Mechanical Engineering, Cleveland, OH 44115 , \\ USA \\ ${ }^{b}$ Motekforce Link, Hogehilweg 18-C,1101 CD Amsterdam, The Netherlands
}

\begin{abstract}
Force plates for human movement analysis provide accurate measurements when mounted rigidly on an inertial reference frame. Large measurement errors occur, however, when the force plate is accelerated, or tilted relative to gravity. This prohibits the use of force plates in human perturbation studies with controlled surface movements, or in conditions where the foundation is moving or not sufficiently rigid. Here we present a linear model to predict the inertial and gravitational artifacts using accelerometer signals. The model is first calibrated with data collected from random movements of the unloaded system and then used to compensate for the errors in another trial. The method was tested experimentally on an instrumented force treadmill capable of dynamic mediolateral translation and sagittal pitch. The compensation was evaluated in five experimental conditions, including platform motions induced by actuators, by motor vibration, and by human ground reaction forces. In the test that included all sources of platform motion, the root-mean-square (RMS) errors were 39.0 $\mathrm{N}$ and $15.3 \mathrm{Nm}$ in force and moment, before compensation, and $1.6 \mathrm{~N}$ and 1.1 $\mathrm{Nm}$, after compensation. A sensitivity analysis was performed to determine the effect on estimating joint moments during human gait. Joint moment errors in hip, knee, and ankle were initially $53.80 \mathrm{Nm}, 32.69 \mathrm{Nm}$, and $19.10 \mathrm{Nm}$, and

\footnotetext{
*Corresponding author

Email addresses: s.hnat@csuohio.edu (Sandra K. Hnat), ben. vanbasten@motekforcelink.com (Ben J. H. van Basten), a.vandenbogert@csuohio.edu (Antonie J. van den Bogert)
}

Preprint submitted to Journal of Biomechanics

April 12, 2019 
reduced to $1.67 \mathrm{Nm}, 1.37 \mathrm{Nm}$, and $1.13 \mathrm{Nm}$ with our method. It was concluded that the compensation method can reduce the inertial and gravitational artifacts to an acceptable level for human gait analysis.

Keywords: biomechanics, instrumentation, gait, inertial artifacts, surface perturbation

Word Count: 3337

\section{Introduction}

Force plates and instrumented treadmills are commonly used to measure ground reaction forces (GRF) for clinical movement analysis, sports performance, or research on human movement. The combination of motion capture and force plate data allow for the calculation of joint moments through inverse dynamic analysis. Recently, instrumented treadmills have become equipped with actuators to translate and rotate the walking surface, for either virtual reality applications or for testing human response to perturbations. Acceleration of the force plate creates large inertial artifacts in the GRF measurement, because a large moving mass is located between the force of interest (foot/ground interface) and the load cells. Additionally, when the frame is tilted, its gravitational mass starts contributing to the horizontal GRF signals. The problem is especially severe in an instrumented treadmill, where the moving mass includes the treadmill frame, motor, and belts. These large errors in GRF data make it impossible to perform standard inverse dynamics in these conditions because the joint moment calculations are based on inaccurate force measurements. The same problem also occurs when the force plate foundation is not sufficiently rigid, or in a moving vehicle in order to study the biomechanics of driving.

Although the problem is noteworthy in human movement analysis, it exists in any load measurement system where the force of interest and the load cells are separated by a moving mass. This includes certain applications in high-speed 
material testing and in force-controlled robots (Hessling, 2009; Dixon, 1990). The work presented in this paper will be applicable in those fields as well.

In principle, the inertial and gravitational forces can be estimated and compensated using rigid body dynamics. This requires knowledge of the mass, inertia matrix, acceleration, angular acceleration, angular velocity, and orientation of the frame. This has been successfully done for one-dimensional linear motion such as in materials testing or a sliding force plate (Hessling, 2009; Dixon, 1990; Pagnacco et al., 2000; Yang and Pai, 2006). While it is straightforward to extend this approach into a six degree of freedom (DOF) load measurement, it becomes impractical due to the requirement to estimate full 3D motion relative to an inertial reference frame, and the use of nonlinear models (Berme and Guler, 2012a,b; Hou et al., 2009; van den Bogert et al., 1996). Furthermore, mass and inertial properties of the frame must be known (Preuss and Fung, 2004). Some of the existing methods neglect the effect of rotation and are limited to compensating for errors due to vibrations within the building or floor (Boschetti et al., 2013).

In this paper, we introduce a simple linear, accelerometer-based compensation method for a fully general inertial and gravitational compensation of force plate data. The linear model is based on the principle that an accelerometer directly measures the inertial and gravitational force on its internal test mass. With a sufficient number of accelerometers, attached at different locations, the total inertial and gravitational artifact of all mass elements in the moving frame will be a linear combination of accelerometer signals (Zappa et al., 2001). The method will be presented and evaluated on an instrumented treadmill in various experimental conditions.

\section{Methods}

\subsection{Compensation Method}

In a 6-DOF load measurement, three-dimensional force and moment are the variables of interest. The compensation model assumes that the effect of gravity 
and inertia on each of the six load signals, when expressed in the local reference frame of the sensor, is a static linear function of $N$ accelerometer signals:

$$
\left[\begin{array}{c}
F_{x} \\
F_{y} \\
F_{z} \\
M_{x} \\
M_{y} \\
M_{z}
\end{array}\right]=C\left[\begin{array}{c}
a_{1} \\
a_{2} \\
\vdots \\
a_{N} \\
1
\end{array}\right],
$$

where $C$ is a $6 \times(N+1)$ matrix of model coefficients. Note that the model includes a constant term for each load variable (the last column of $C$ ) which will be used by the calibration to remove any static offsets that may be present in the load cell signals. It is not necessary to calibrate the accelerometers. Neither is it necessary to separate the accelerometer information into acceleration and gravitational effects. In fact, it is essential that such a separation is not performed because inertial artifacts arise from the combination of the two (van den Bogert et al., 1996). Raw accelerometer signals should be used.

The model must be calibrated by a system identification experiment, in which no external load is applied, and the load cells will only measure the artifacts. This is best done with random movements of the frame, to explore the entire space of potential artifacts. During this experiment, $K$ samples of force and moment data are collected, along with accelerometer data. First, the data is arranged into matrices $A$ (accelerometer signals) and $F$ (force and moment data):

$$
\begin{gathered}
A=\left[\begin{array}{ccccc}
a_{11} & a_{12} & \cdots & a_{1 N} & 1 \\
\vdots & \vdots & \vdots & & \vdots \\
a_{K 1} & a_{K 2} & \cdots & a_{K N} & 1
\end{array}\right] \\
F=\left[\begin{array}{cccccc}
F_{1 x} & F_{1 y} & F_{1 z} & M_{1 x} & M_{1 y} & M_{1 z} \\
\vdots & \vdots & \vdots & \vdots & \vdots & \vdots \\
F_{K x} & F_{K y} & F_{K z} & M_{K x} & M_{K y} & M_{K z}
\end{array}\right]
\end{gathered}
$$


and the model coefficients $C$ are determined through a least-squares solution of the overdetermined system of linear equations:

$$
A C^{T}=F
$$

The calibration was coded in Matlab (version 2016a) as well as in $\mathrm{C}++$. In Matlab, QR decomposition was used to obtain the least-squares solution, which is implemented as the "backslash" operator:

$$
C=(A \backslash F)^{\prime}
$$

In $\mathrm{C}++$, model coefficients were determined through linear regression using the Shark library (Igel et al., 2008). It was verified that the results were identical in both implementations.

\subsection{Experimental Validation}

Experiments were performed on a split-belt instrumented treadmill capable of actuated mediolateral translation (sway) along the $\mathrm{X}$-axis, and sagittal pitch in the YZ-plane (V-Gait, Motekforce Link, Amsterdam, Figure 1). Each belt assembly, including motors, has a mass of about $150 \mathrm{~kg}$ which is located between the load cells and the walking surface. Two triaxial accelerometers (4030 2G range, Measurement Specialties) were mounted on the side of the treadmill (posterior-left and anterior-right), separated by $1.30 \mathrm{~m}$ in both the lateral and anterior/posterior directions (Euclidean distance of $1.83 \mathrm{~m}$ ). Zappa (Zappa et al., 2001) proved that four non-coplanar accelerometers are sufficient to uniquely determine the acceleration at each point in a rigid body. Since there are only two actuated degrees of freedom (DOF, pitch and sway) in this application, only two accelerometers are required to detect the range of movements.

Sway and pitch signals were commanded to the treadmill using D-Flow 3.24 (Motekforce Link, Amsterdam). GRFs from the right belt, and accelerometer data, were directly acquired in the data acquisition unit of an optical motion capture system (Nexus 1.8.5, Vicon). All files were exported in C3D files for further offline analysis. Sampling rate was $1000 \mathrm{~Hz}$. All signals were filtered 
with a $2^{\text {nd }}$ order low-pass Butterworth filter with a cut-off frequency of $6 \mathrm{~Hz}$, which is typically used for inverse dynamic analysis of walking (Winter, 1990; van den Bogert et al., 2013). After filtering, the first full second of data was removed to eliminate the filter startup effect.

The $6 \times 7$ model coefficient matrix $C$ was calibrated using a 60 -second unloaded trial in which the treadmill surface was randomly translated and rotated. Zero-mean Gaussian white noise signals were generated with a sampling time of $3.3 \mathrm{~ms}$ and RMS amplitudes of $0.707 \mathrm{~m} / \mathrm{s}^{2}$ and $127 \% \mathrm{~s}^{2}$ for sway and pitch, respectively, in MATLAB Simulink (Mathworks, Natick, MA, USA), as shown in Figure 2. The signals were twice-integrated to obtain smooth treadmill displacements with realizable accelerations. Integration drift was eliminated by processing the input signals through high-pass filters ( $2^{\text {nd }}$ order Butterworth) with a passband edge frequency of $0.21 \mathrm{~Hz}$. Upper and lower limits were imposed on the signals, thereby restricting the translation and rotation movements to the maximum of $\pm 0.05 \mathrm{~m}$ displacement and $\pm 10^{\circ}$ pitch angle.

The calibrated model was evaluated in five tests where the platform was moved without load applied to the right force plate. The tests included platform motions induced by actuators, by motor vibration, and by human ground reaction force impacts. In Trial 1 , the treadmill was subjected to 60 seconds of random pitch and sway movement generated by the same procedure as the calibration trial, but with a different random number seed. Trial 2 had the same random movement, but with the treadmill belts running at $1.3 \mathrm{~m} / \mathrm{s}$ to evaluate the effect of motor vibration. Trial 3 again contains the same random movement as Trial 1 and 2 , but with a subject walking on the left belt at $1.3 \mathrm{~m} / \mathrm{s}$. In Trial 4, the subject walked on the left belt with $1.3 \mathrm{~m} / \mathrm{s}$, without pitch or sway motion. In this trial, the subject walked on the front half of the treadmill to represent the worst case of walking-induced frame motion. In Trial 5 , the treadmill was set in a static pitch angle of 9 degrees. As the treadmill is not moving in this test, all artifacts are induced by gravity.

In each trial, the model was used to predict the inertial and gravitational force and moment artifacts from the accelerometer signals. The predicted arti- 
facts were subtracted from the measured forces and moments. Since no external loads were applied to the right force plate, the resulting forces and moments would be zero if the compensation was perfect. To quantify the performance of the compensation method, we determine the RMS (root-mean-square) of the force and moment residuals. This was done on all six load variables, on all test trials, before filtering, after filtering, and after the compensation. This allowed us to quantify how much reduction in the artifacts was achieved by filtering alone, and how much additional reduction was achieved by the linear compensation model.

$$
\mathrm{RMS}=\sqrt{\frac{\sum\left(F_{\text {estimate }}-F_{\text {measured }}\right)^{2}}{n}}
$$

where $F_{\text {estimate }}=A_{\text {measured }} C^{T}$ and $A_{\text {measured }}$ are the measured accelerometer signals.

\subsection{Sensitivity Analysis}

To assess the importance of inertial and gravitational artifacts and the effectiveness of the compensation, we used human gait data that was collected on a stationary instrumented treadmill where the artifacts were negligible (Moore et al., 2015). A standard sagittal plane inverse dynamic analysis was performed (Winter, 1990) three times. First, with the original "true" GRF data. Second, with inertial artifacts from Trial 3 added to the original GRF. Third, with the inertial artifacts from Trial 3 , after compensation using the previously calibrated model, added to the original GRF data. Trial 3 was chosen because it is the test condition that includes all sources of platform motion, leading to the highest inertial artifact. No low-pass filtering was applied to the inertial artifacts and the accelerometer data that was used to compensate. However, the subsequent inverse dynamic analysis used a $6 \mathrm{~Hz}$ low-pass filter for both kinematic and force plate data (van den Bogert et al., 2013). 


\section{Results}

Figure 3 illustrates the performance of the compensation method on the first four seconds of Trial 3 which contains artifacts due to actuated random motions, human ground reaction forces, and motor vibration. Before compensation, there are large artifacts, especially in $F_{x}, F_{z}, M_{x}$, and $M_{y}$. The artifacts in $F_{z}$ and $M_{y}$ are mostly gravitational and reflect low-frequency changes in pitch angle. The inertial contributions have higher frequencies, reflecting the sensitivity to linear and angular accelerations, rather than orientation. After predicting the artifacts from the accelerometer signals, and subtracting them from the measured loads, the remaining artifact is small.

For each trial, the effectiveness of the compensation was quantified by the RMS comparison between uncompensated and compensated force signals. Results for the 5 conditions are shown in Table 1.

The largest inertial artifacts were present in Trial 3. When residuals in all coordinate axes were combined, the overall RMS errors were $39.0 \mathrm{~N}$ and 15.3 $\mathrm{Nm}$ in force and moment components, respectively, after filtering. The compensation reduced these errors to $1.6 \mathrm{~N}$ and $1.1 \mathrm{~N} \mathrm{~m}$. The differences between the trials provide insight into the effect of the various mechanical inputs. The errors after compensation were lowest when the platform motion was entirely induced by the actuators (Trial 1), and increased due to vibrations caused by the motors that drive the belts (Trial 2). There was a slight further increase in Trial 3, when human ground reaction forces were applied to the moving frame. In Trial 4, platform motions were solely induced by human ground reaction forces and motor vibration. In case of a static pitch of 9 degrees (Trial 5) the gravitational effects were large and consistent with the downhill component of the gravitational force on the $150 \mathrm{~kg}$ platform mass. After compensation, these errors were reduced to less than $1 \mathrm{~N}$ and $1 \mathrm{Nm}$. Even in the most challenging test cases (Trials $2,3,4$ ), the compensation reduced the artifacts to values below $2 \mathrm{~N}$ and $2 \mathrm{~N} \mathrm{~m}$.

Figure 4 shows the human joint moments that would be obtained if the GRF 
data had been affected by the inertial and gravitational artifacts from Trial 3 . The joint moments were greatly affected, mostly by the horizontal force $F_{z}$ and the effect was largest in the hip joint. Before compensation, the RMS errors in hip, knee, and ankle moments were $53.80 \mathrm{~N} \mathrm{~m}, 32.69 \mathrm{~N} \mathrm{~m}$, and $19.10 \mathrm{~N} \mathrm{~m}$. After compensation, these errors were reduced to $1.67 \mathrm{Nm}, 1.37 \mathrm{Nm}$, and $1.13 \mathrm{Nm}$, respectively.

\section{Discussion}

Rotating and translating a platform equipped with load cells will introduce inertial artifacts in the force measurements. We developed a linear model to estimate these errors from accelerometers so that these errors can be compensated. The method was evaluated on the test case of human gait analysis on an instrumented treadmill undergoing movement. It was found that the errors were initially too large to allow a useful inverse dynamic gait analysis. The compensation method reduced the errors to acceptable levels within the range of noise typically observed in force plate signals. This makes it possible to study the dynamics and control of human gait during mechanical perturbations. This was previously only attempted for simple platform translations or rotations (Dixon, 1990; Pagnacco et al., 2000; Preuss and Fung, 2004). Our method does not require measuring the kinematics of the moving frame relative to an inertial reference frame and does not require knowledge of the mass properties of the frame, nor exact locations of the accelerometers. Furthermore, our residual forces and moments were far lower than those obtained on an instrumented treadmill with the existing methods (Preuss and Fung, 2004).

A similar accelerometer-based method was described for an application where load cells were used to determine the location, where a computer screen was touched (Roberts, 2006). In our application, the mass of the moving frame was, however, much larger. With a mass of $150 \mathrm{~kg}$, an acceleration of only $0.01 \mathrm{~m} \mathrm{~s}^{-2}$ $(1 \mathrm{mg})$ is sufficient to induce an inertial artifact of $1.5 \mathrm{~N}$. Reducing the artifact to this level required accelerometers with sufficient accuracy and resolution. 
Tests were performed on a system with two actuated DOFs (pitch and sway), but the compensation method is completely general and can compensate for artifacts due to full 6-DOF motions in all six load components, using any number of accelerometer signals. To reliably detect inertial artifacts during arbitrary 3D translations and rotations, more than two triaxial accelerometers should be used. With only two accelerometers, an angular velocity or acceleration about an axis through the two accelerometers cannot be detected. Zappa's method (Zappa et al., 2001) used four non-coplanar accelerometers, but did not show that this was necessary. It may be possible to use only three accelerometers, or a coplanar accelerometer configuration. Because our method uses a least-squares approach, any number of accelerometers can be used, and more accelerometers will give better results. The number of accelerometers should be selected based on the DOFs of the particular system. Including more accelerometers will more effectively reduce noise and non-rigid effects, though these additions will eventually have diminishing returns.

Another attractive feature of the approach is that the calibration matrix automatically includes the linear transformations that are needed to predict forces and moments in the force plate coordinate system. Therefore, the accelerometers can be randomly oriented and measure data in their own coordinate system.

The compensation method is based on the assumption that the moving platform is a rigid body. Inertial artifacts associated with deformation or vibration within the frame will only be partially compensated. This explains why our results were slightly worse in the trials where internal vibration was introduced from the belt motors or human impacts. However, it may be possible to use a larger array of accelerometers to detect and compensate for non-rigid modes. Another possibility is to use and calibrate a dynamic model that can simulate vibrations. Treadmill frames have internal moving parts (motor and rollers) which will introduce additional artifacts if their speed is not constant. This artifact only exists in the sagittal plane moment $M_{x}$. A dynamic, 1-DOF compensation model was successfully developed (Hnat and van den Bogert, 2014) and can be added on to the techniques presented here. 
During all tests, signals were low-pass filtered at $6 \mathrm{~Hz}$ which is typical for human gait analysis. When higher bandwidth is needed, the remaining error will be larger because more of the non-rigid motion effect will be included. Therefore, the performance of the compensation method must be tested for a specific application and bandwidth requirement.

In one of the test conditions (Trial 4), the frame was not actively moved, but all movements were due to the motors and human GRF. The pitch moment $\left(M_{x}\right)$ artifact was about $10 \mathrm{Nm}$, after filtering, due to a small rotational vibration about the pitch axis. Such a vibration is expected based on the structure of the machine (Figure 1). The frequency of this vibration was low enough that the artifact was not sufficiently attenuated by the $6 \mathrm{~Hz}$ low pass filter alone. However, the accelerometer-based compensation reduced the error to $1.04 \mathrm{Nm}$ which is acceptable for gait analysis. Furthermore, as our compensation method consists of a single matrix multiplication (and vector subtraction), it can be applied in real-time in combination with other real-time biomechanical models (van den Bogert et al., 2013).

Traditionally, force measuring systems are designed with a natural frequency that is well above the frequency of interest. Our results demonstrate that this requirement can be relaxed, because low-frequency errors can be partially removed by inertial compensation. Similarly, artifacts from low frequency building vibrations, or from doing force measurement in moving vehicles, could be compensated. This reduces the need to place force measuring systems in basements or on a separate mechanically isolated foundation. Because accelerometers are inexpensive, and the compensation method is easily implemented, it may be wise to include inertial compensation as a standard feature on force measuring systems that are sensitive to inertial artifacts.

Load cells measure forces and moments in the local (moving) reference frame, and the compensation is carried out entirely in that reference frame. When load measurement is combined with motion capture performed in an inertial reference frame, a coordinate transformation is needed to bring the (compensated) load data into the inertial reference frame. This transformation requires knowledge of 
the position and orientation of the moving frame. In the actuated treadmill, the position and orientation of the frame is known from the encoders in the position control system. Alternatively, optical motion capture can be used, with three or more motion capture markers placed on the treadmill frame (Challis, 1995).

\section{Conclusion}

The proposed method is capable of reducing inertial and gravitational artifacts in data obtained from a moving instrumented treadmill. For gait applications, the remaining error is small enough to allow accurate joint torque calculations through inverse dynamic analysis.

\section{Acknowledgment}

This research was supported by the National Science Foundation under Grant No. 1344954 and by the Ohio Department of Development, Third Frontier Commission. The authors thank Co van Vliet for mounting and wiring the accelerometers.

\section{Conflict of Interest Statement}

There are no conflicts of interest for any of the authors regarding the research reported in this manuscript.

\section{References}

Antonsson, E. K., Mann, R. W., 1985. The frequency content of gait. Journal of Biomechanics 18 (1), $39-47$.

Berme, N., Guler, H., 2012a. Force and/or motion measurement system having inertial compensation and method thereof. US Patent $8,315,823$.

Berme, N., Guler, H., 2012b. Force measurement system having inertial compensation. US Patent $8,315,822$. 
Boschetti, G., Caracciolo, R., Richiedei, D., Trevisani, A., 2013. Model-based dynamic compensation of load cell response in weighing machines affected by environmental vibrations. Mechanical Systems and Signal Processing 34 (1-2), $116-130$.

Cappello, A., Palombara, P. F. L., Leardini, A., 1996. Optimization and smoothing techniques in movement analysis. International Journal of Bio-Medical Computing 41 (3), $137-151$.

Challis, J. H., 1995. A procedure for determining rigid body transformation parameters. Journal of Biomechanics 28 (6), $733-737$.

Dixon, M. J., 1990. Development of a load-cell compensation system. Experimental Mechanics 31, $21-24$.

Hamill, J. K. K. M., 2008. Biomechanical Basis of Human Movement, 3rd Edition. Lippincott Williams and Wilkins.

Hessling, J., 2009. Models of dynamic measurement error variations of material testing machines. Mechanical Systems and Signal Processing 23 (8), $2510-$ 2518.

Hnat, S. K., van den Bogert, A. J., 2014. Inertial compensation for belt acceleration in an instrumented treadmill. Journal of Biomechanics 47 (15), 3758 $-3761$.

Hou, Z. C., Lu, Y. N., Lao, Y. X., Liu, D., 2009. A new trifilar pendulum approach to identify all inertia parameters of a rigid body or assembly. Mechanism and Machine Theory 44 (6), $1270-1280$.

Igel, C., Heidrich-Meisner, V., Glasmachers, T., 2008. Shark 2.1. Journal of Machine Learning Research 9, 993-996.

Kirtley, C., 2006. ClinicalGait Analysis: Theory and Practice, 3rd Edition. Elsevier Health Sciencies. 
Kristianslund, E., Krosshaug, T., van den Bogert, A. J., 2012. Effect of low pass filtering on joint moments from inverse dynamics: Implications for injury prevention. Journal of Biomechanics 45 (4), $666-671$.

Lohr, R., Hayford, P., Bahra, C., Jan. 21 2003. Dynamic load cell apparatus. US Patent 6,508,132.

MATLAB, 2014. version 8.3.0.532 (R2014a). The MathWorks Inc., Natick, Massachusetts.

McLean, S. G., Huang, X., van den Bogert, A. J., 2005. Association between lower extremity posture at contact and peak knee valgus moment during sidestepping: Implications for acl injury. Clinical Biomechanics 20 (8), 863 870 .

Moore, J. K., Hnat, S. K., van den Bogert, A. J., 2015. An elaborate data set on human gait and the effect of mechanical perturbations. PeerJ 3, e918.

Pagnacco, G., Silva, A., Oggero, E., Berme, N., 2000. Inertially compensated force plate: a means for quantifying subject's ground reaction forces in noninertial conditions. Biomedical Sciences Instrumentation 36, $397-402$.

Preuss, R., Fung, J., 2004. A simple method to estimate force plate inertial components in a moving surface. Journal of Biomechanics 37 (8), 1177 1180.

Roberts, J., 2006. Force measurement system correcting for inertial interference. US Patent 7,102,621.

Soderkvist, I., Wedin, P.-A., 1993. Determining the movements of the skeleton using well-configured markers. Journal of Biomechanics 26 (12), 1473 - 1477.

van den Bogert, A. J., de Koning, J., 1996. On optimal filtering for inverse dynamic analysis. In: Proceedings of the IXth Biennial Conference of the Canadian Society for Biomechanics. 
van den Bogert, A. J., Geijtenbeek, T., Even-Zohar, O., Steenbrink, F., Hardin, E. C., 2013. A real-time system for biomechanical analysis of human movement and muscle function. Medical and Biological Engineering and Computing $10,1069-1077$.

van den Bogert, A. J., Read, L., Nigg, B. M., 1996. A method for inverse dynamic analysis using accelerometry. Journal of Biomechanics 29 (7), $949-$ 954.

Winter, D. A., 1990. Biomechanics and motor control of human movement, 2nd Edition. J Wiley and Sons.

Yang, F., Pai, Y.-C., 2006. Correction of the inertial effect resulting from a plate moving under low-friction conditions. Journal of Biomechanics 40, 2723-2730.

Zappa, B., Legnani, G., van den Bogert, A. J., Adamini, R., 2001. On the number and placement of accelerometers for angular velocity and acceleration determination. Journal of Dynamic Systems, Measurement, and Control 123, $552-554$. 


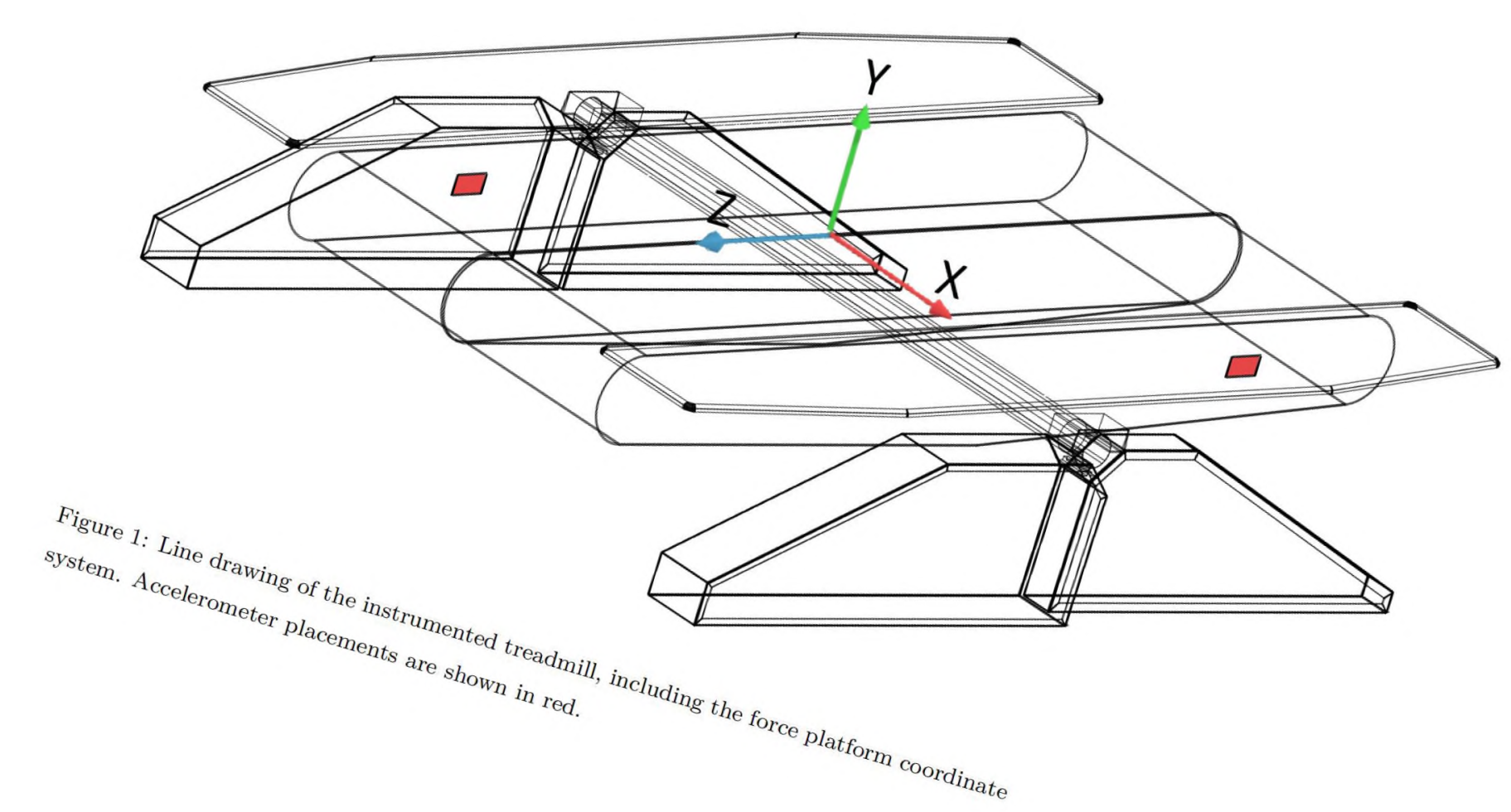



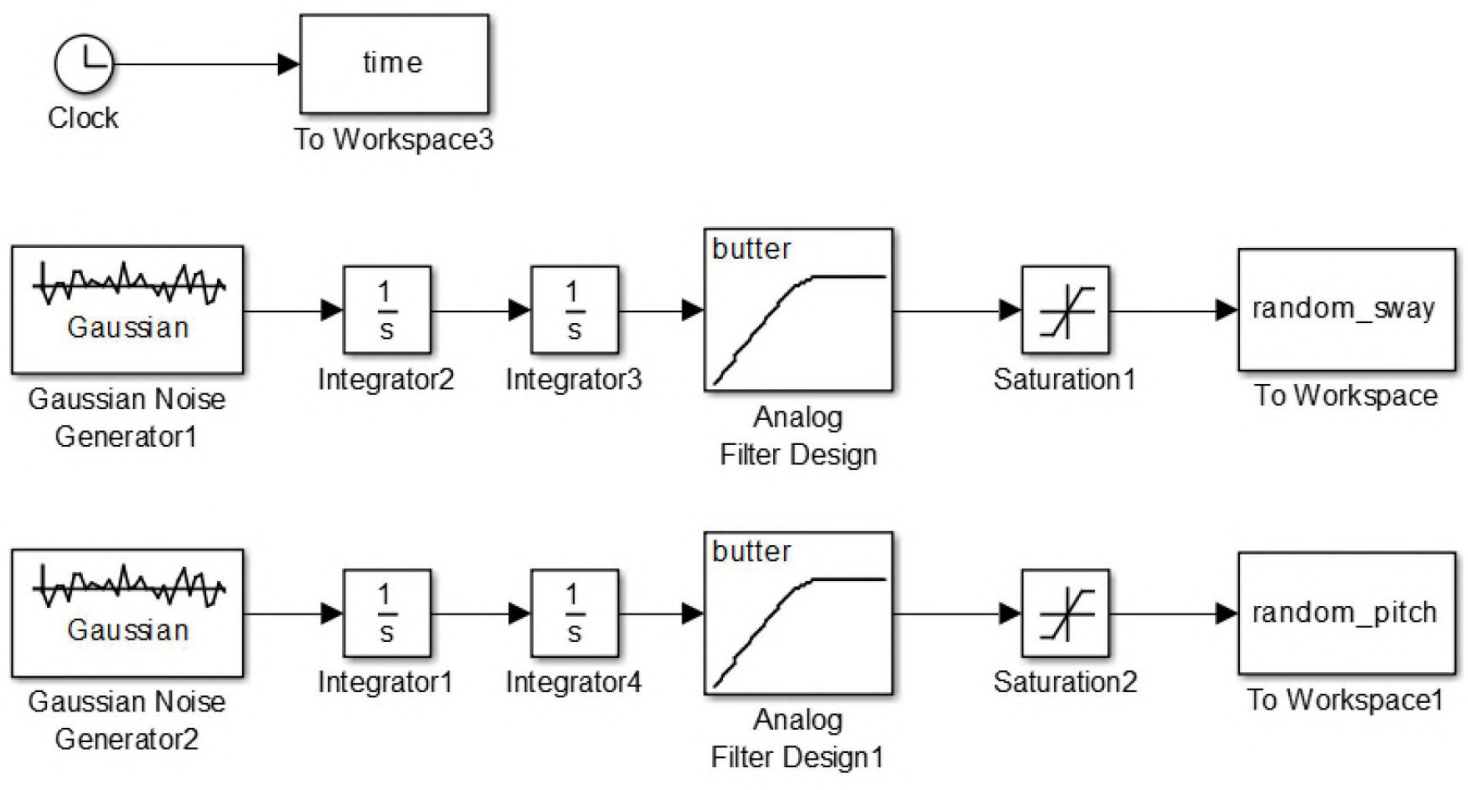

Figure 2: MATLAB Simulink diagram for generating random sway (mediolateral translation) and sagittal pitch (rotation) commands 

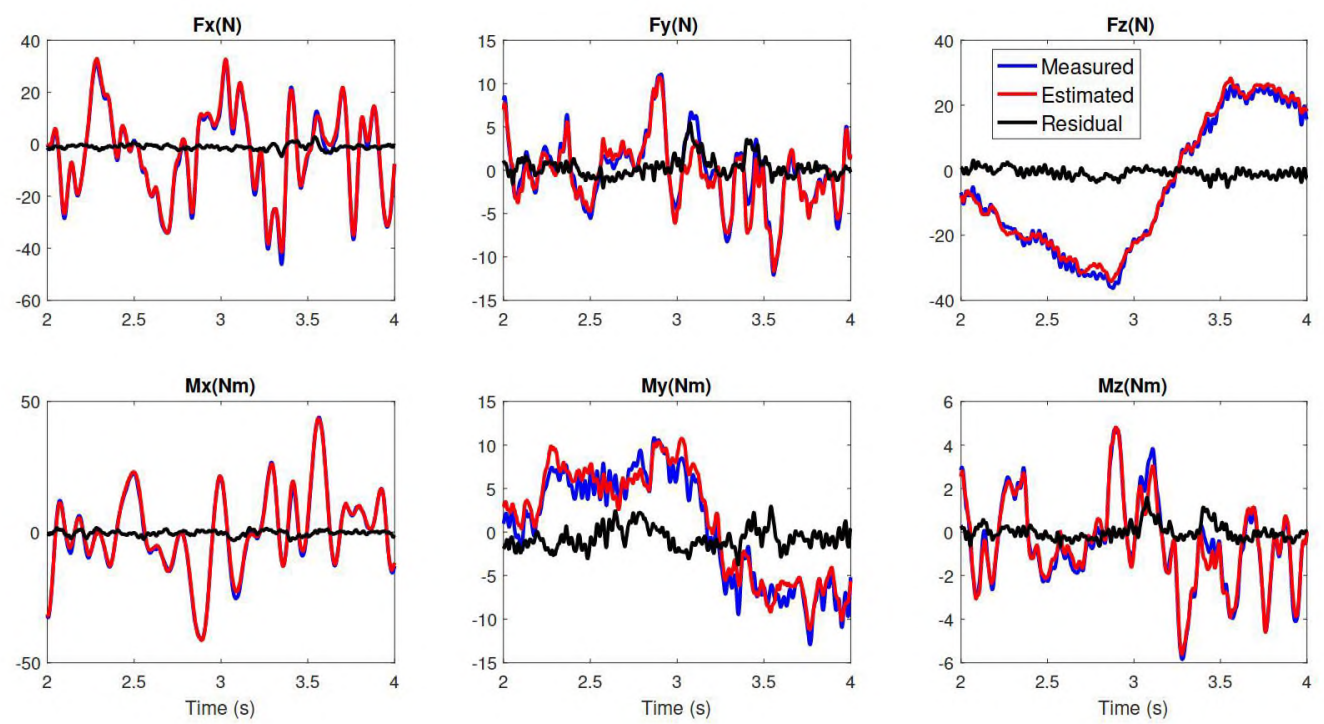

Figure 3: Force and moment data from Trial 3. The measured inertial artifact (blue) was compared to the prediction from accelerometers (red). The residuals (black) are the errors that remain after subtracting the predicted inertial artifact. All data were $6 \mathrm{~Hz}$ low-pass filtered before processing. Note the different scales on the vertical axes. 

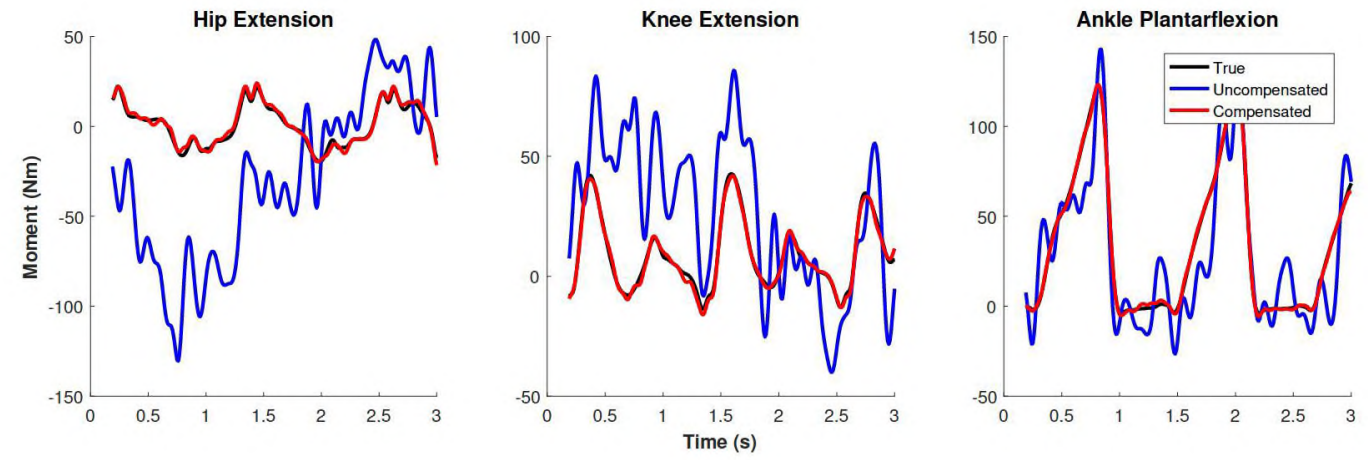

Figure 4: Hip, knee, and ankle joint moments during walking, affected by the uncompensated (blue) and compensated (red) inertial artifacts from Trial 3. The compensated result is almost identical to the "true" result from the original data containing no platform movement (black). 


\begin{tabular}{|c|c|c|c|c|}
\hline Test condition & Variable & RMS(raw) & RMS(filtered) & RMS(compensated) \\
\hline \multirow{6}{*}{$\begin{array}{l}\text { Trial 1: random } \\
\text { pitch and sway }\end{array}$} & $F_{x}(\mathrm{~N})$ & 35.32 & 16.13 & 0.57 \\
\hline & $F_{y}(\mathrm{~N})$ & 9.99 & 3.91 & 0.76 \\
\hline & $F_{z}(\mathrm{~N})$ & 69.62 & 65.69 & 0.89 \\
\hline & $M_{x}(\mathrm{~N} \mathrm{~m})$ & 18.54 & 13.18 & 0.76 \\
\hline & $M_{y}(\mathrm{Nm})$ & 24.82 & 19.07 & 0.64 \\
\hline & $M_{z}(\mathrm{Nm})$ & 4.50 & 2.04 & 0.23 \\
\hline \multirow{6}{*}{$\begin{array}{l}\text { Trial 2: random pitch and } \\
\text { sway, } 1.3 \mathrm{~m} / \mathrm{s} \text { belt speed }\end{array}$} & $F_{x}(\mathrm{~N})$ & 33.17 & 16.02 & 1.28 \\
\hline & $F_{y}(\mathrm{~N})$ & 11.61 & 3.91 & 1.17 \\
\hline & $F_{z}(\mathrm{~N})$ & 69.08 & 65.72 & 1.52 \\
\hline & $M_{x}(\mathrm{Nm})$ & 18.75 & 13.24 & 1.16 \\
\hline & $M_{y}(\mathrm{Nm})$ & 24.54 & 19.04 & 1.25 \\
\hline & $M_{z}(\mathrm{~N} \mathrm{~m})$ & 4.49 & 2.05 & 0.35 \\
\hline \multirow{6}{*}{$\begin{array}{l}\text { Trial 3: random pitch } \\
\text { and sway, } 1.3 \mathrm{~m} / \mathrm{s} \text { belt } \\
\text { speed, human walking }\end{array}$} & $F_{x}(\mathrm{~N})$ & 33.69 & 16.16 & 1.51 \\
\hline & $F_{y}(\mathrm{~N})$ & 20.25 & 5.19 & 1.22 \\
\hline & $F_{z}(\mathrm{~N})$ & 71.45 & 65.41 & 1.88 \\
\hline & $M_{x}(\mathrm{~N} \mathrm{~m})$ & 26.11 & 18.42 & 1.24 \\
\hline & $M_{y}(\mathrm{Nm})$ & 26.56 & 18.91 & 1.46 \\
\hline & $M_{z}(\mathrm{Nm})$ & 6.89 & 2.43 & 0.38 \\
\hline \multirow{6}{*}{$\begin{array}{l}\text { Trial } 4: 1.3 \mathrm{~m} / \mathrm{s} \text { belt } \\
\text { speed, human walking }\end{array}$} & $F_{x}(\mathrm{~N})$ & 12.29 & 1.31 & 1.44 \\
\hline & $F_{y}(\mathrm{~N})$ & 13.94 & 2.75 & 1.06 \\
\hline & $F_{z}(\mathrm{~N})$ & 17.89 & 1.53 & 1.63 \\
\hline & $M_{x}(\mathrm{Nm})$ & 18.68 & 9.65 & 1.04 \\
\hline & $M_{y}(\mathrm{Nm})$ & 11.68 & 1.28 & 1.33 \\
\hline & $M_{z}(\mathrm{Nm})$ & 4.43 & 1.07 & 0.32 \\
\hline \multirow{6}{*}{ Trial 5: $9^{\circ}$ static pitch } & $F_{x}(\mathrm{~N})$ & 1.65 & 1.44 & 0.42 \\
\hline & $F_{y}(\mathrm{~N})$ & 19.46 & 19.44 & 0.96 \\
\hline & $F_{z}(\mathrm{~N})$ & 227.33 & 227.33 & 0.66 \\
\hline & $M_{x}(\mathrm{Nm})$ & 24.87 & 24.86 & 0.39 \\
\hline & $M_{y}(\mathrm{Nm})$ & 65.08 & 65.07 & 0.38 \\
\hline & $M_{z}(\mathrm{Nm})$ & 6.20 & 6.19 & 0.33 \\
\hline
\end{tabular}

Table 1: Root-mean-square (RMS) values of force and moment data from the unloaded force plate during the test trials. See text for details. 